\title{
Fabrication of Fiber Filters with Antibacterial Properties for VOC and Particle Removal
}

\author{
Paxton Juuti $^{1^{*}}$, Markus Nikka ${ }^{1}$, Marianne Gunell ${ }^{2,3}$, Erkki Eerola ${ }^{2,3}$ Jarkko J. Saarinen $^{4}$, \\ Youichi Omori $^{5}$, Takafumi Seto ${ }^{6}$, Jyrki M. Mäkelä ${ }^{1}$ \\ ${ }^{1}$ Aerosol Physics, Faculty of Engineering and Natural Sciences, Tampere University, 33720 Tampere, Finland \\ ${ }^{2}$ Department of Clinical Microbiology, Turku University Hospital, 20521 Turku, Finland \\ ${ }^{3}$ Department of Medical Microbiology and Immunology, University of Turku, 20500 Turku, Finland \\ ${ }^{4}$ Department of Chemistry, University of Eastern Finland, Joensuu, Finland \\ ${ }^{5}$ Sanzen Seishi Co. Ltd., Kanazawa, Ishikawa 920-0338, Japan \\ ${ }^{6}$ School of Natural System, College of Science and Engineering, Kanazawa University, Kanazawa, Ishikawa 920-1192, \\ Japan
}

\begin{abstract}
The use of filters to control air quality has been implemented widely in all types of structures. Unfortunately, filters risk becoming platforms for the growth of bacteria, which can then be dispersed further in the air stream. To combat this, antibacterial materials are being incorporated into filter media. In this work, we tested two routes for introducing nanoscale silver into filters containing activated carbon fibers (ACF): first, by adding silver nanofibers directly to the fiber fabrication process and second, by coating a pre-existing filter with silver nanoparticles generated by a liquid flame spray (LFS). The resultant filters were evaluated for methanol adsorption, particle penetration and antibacterial activity. The results show that both methods are suitable for producing antibacterial filters as well as being highly tailorable and scalable for specific needs.
\end{abstract}

Keywords: Air filtration; Liquid flame spray; Nanoscale silver; Adsorption.

\section{INTRODUCTION}

Indoor air quality has an important role on human health (Jones, 1999), and, worryingly, its impacts can be seen in institutes such as schools (Daisey et al., 2003) and hospitals (Saad, 2002). One way to improve the quality of indoor air is to utilize filtration in the air conditioning system. However, filters are susceptible to becoming growing platforms for microbes, especially in humid conditions (Möritz et al., 2001; Forthomme et al., 2014). Furthermore, biocompatible carbon-based adsorptive materials can also be used if volatile organic compounds (VOCs), such as formaldehyde (Rumchev et al., 2002), are desired to be filtered from air alongside with the particulate matter. Unfortunately, due to their good biocompatibility, carbonbased materials could make the filters more susceptible to microbes (Yoon et al., 2008). To combat these problems, antimicrobial materials have been widely adopted in material

\footnotetext{
* Corresponding author.

Tel.: +358401981026

E-mail address: paxton.juuti@tuni.fi
}

functionalization. Arguably, the most widely used material for this purpose currently is silver (Oya et al., 1993; Jung et al., 2011; Brobbey et al., 2017), but also metal oxides, such as zinc oxide (Gordon et al., 2011) and copper oxide (Hassan et al., 2014), are being utilized. In humid conditions where bacterial growth might pose a risk, the use of silver nanoparticles should be very effective against bacterial growth, due to the transfer of silver ions and whole nanoparticles, which can disrupt the vital functions of the bacterial cells (Sotiriou and Pratsinis, 2010).

Here, we take a closer look at fabricating fiber filters containing activated carbon fibers (ACF) and adding antibacterial silver in two different morphologies: (1) fibers and (2) particles. The silver fibers will be added during the filter fabrication process, whereas the particles will be added to an already fabricated filter as a coating. For the nanoparticle coating, the liquid flame spray (LFS; Tikkanen et al., 1994; Mäkelä et al., 2017) method will be implemented. It has been used quite extensively for introducing nanoparticle coatings on a wide range of materials, most recently on paper (Haapanen et al., 2018), glass (Teisala et al., 2018) and plastic (Juuti et al., 2017). The LFS method can produce other metal and metal-oxide particles and is readily scalable, so the process investigated here has 
potential for future antibacterial materials as well. In essence, we introduce a scalable and tailorable approach for fabricating filters containing fibrous and particulate nanoscale silver and compare their antibacterial activities against Staphylococcus aureus (S. aureus) and Escherichia coli $(E$. coli). Furthermore, the filters will be tested for particle penetration and methanol adsorption capability to ascertain their functionality as a proper and usable filtration medium.

\section{MATERIALS AND METHODS}

\section{Filter Media Fabrication}

Three different kinds of fiber filter media were produced by mixing known mass ratios (see Table 1) of select fiber materials. The fiber filters were prepared by first premixing the appropriate amount of micro- and nanofibers into 500 $\mathrm{cm}^{3}$ of water to make a $0.28 \%$ slurry, which was then mixed for $2 \mathrm{~min}$ at $3000 \mathrm{rpm}$. The premixed slurry was then diluted to $0.019 \%$ in paper processing equipment by introducing an additional $7000 \mathrm{~cm}^{3}$ of water. To make the filter discs, the slurry was filtered with mesh, leaving a thin filter medium on top. Lastly, to remove the residual water from the filter structure, the filter discs were dried at $80^{\circ} \mathrm{C}$ for $25 \mathrm{~min}$. The three prepared filter media all have the same base composition of glass fibers, binding polyvinyl alcohol (PVA) fibers and activated carbon fibers (ACF), with diameters of $10.5,10$ and $18 \mu \mathrm{m}$ respectively. The second and third filter media get an addition of $\sim 300 \mathrm{~nm}$ glass nanofibers in the fiber mixture. Lastly, only the third filter medium has $1 \mathrm{~m}-\%$ of silver nanofibers (30 nm; Sanzen Seishi Co., Ltd.) substituting equal mass of the glass nanofibers. These filter media have the following denotations: $\mathrm{F} 0$ is the microfiber filter, F1 is the glass nanofiber filter and F2 is the silver nanofiber filter, noting that the addition of a different fiber material is cumulative through the series, which can be seen clearly from Table 1 .

\section{Coating of the Filter Media}

Another route for adding the nanoscale silver to the filter media was utilized by coating an already prepared F1 filter media with the LFS method. The coating setup can be seen in Fig. 1(a). The LFS produced nanoscale silver from an aqueous silver nitrate $\left(\mathrm{AgNO}_{3}\right)$ precursor (silver nitrate, ACS, 99.9+\% [metals basis]; Alfa Aesar) solution with a concentration of $125 \mathrm{mg} \mathrm{mL}^{-1}$ of pure silver, which was fed with a flow rate of $4 \mathrm{~mL} \mathrm{~min}{ }^{-1}$. The precursor was thermally decomposed and evaporated in a hydrogen-oxygen flame with a gas-ratio of 20 to $10 \mathrm{~L} \mathrm{~min}^{-1}$, respectively, with an additional collar flow of $5 \mathrm{~L} \mathrm{~min}^{-1}$ of nitrogen. The silver condensed and the final particle shape was formed fully in the following residence tube (Sorvali et al., 2017), from where it was sampled with an inlet connected to an ejector diluter (ED), further diluted and cooled, and guided to be deposited on the filter media. A similar synthesis protocol was implemented recently in the work of Harra et al. (2017) for silver and aluminum oxide. This produced the fourth filter medium studied in this work and is denoted as F3, and the mass fractions of its constituent fibers can be seen in Table 1 .

For comparable results, the mass loading of the deposited silver coating was kept constant between the as-prepared silver nanofiber filter and the silver-coated filter. To achieve this, the measurement of the process is required in addition to the penetration characteristics of the filter being coated, which is discussed in the next chapter. The instruments require additional dilution of the sample, so an additional ejector diluter was used. The number size distribution of the nanoscale silver was measured using a Scanning Mobility Particle Sizer (SMPS; Model 3938; TSI Inc.), which was paired with the aerodynamic sizing of an Electrical Low Pressure Impactor (ELPI+; Dekati) in order to get additional information on the effective density $\left(\rho_{\text {eff }}\right)$. The effective density can be calculated using the following equation (Kulkarni et al., 2011):

$$
\rho_{e f f}=\rho_{0} \frac{C_{c}\left(d_{a}\right) d_{a}^{2}}{C_{c}\left(d_{b}\right) d_{b}^{2}},
$$

where $\rho_{0}$ is the unit density, $C_{c}$ is the slip correction factor, and $d_{b}$ and $d_{a}$ are mobility and aerodynamic diameters measured by SMPS and ELPI+, respectively. For real-time monitoring purposes, DENSMO (Juuti et al., 2016) was used in parallel with the previous instruments. It also utilizes Eq. (1) for characterizing the effective density. DENSMO operates based on the series measurement of mobility median and aerodynamic median diameters. The measurements are done electrically, so the aerosol particles are first charged with a corona charger.

For mass characterization, a Tapered Element Oscillating Microbalance (TEOM; Rupprecht \& Patashnick Co., Inc.) and a Quartz Crystal Microbalance Micro-Orifice Uniform Deposition Impactor (QCM-MOUDI; TSI Inc.) were used for measuring the total mass concentration and the mass-size distribution, respectively. Additional estimation of the

Table 1. Composition of fabricated filters.

\begin{tabular}{llllll}
\hline & Microfibers & Nanofibers & Ag-fibers & Ag-particles \\
& F0 & F1 & F2 & F3 & \\
\hline Glass fibers & 60 & 50 & 50 & 49.5 & $\mathrm{~m}-\%$ \\
PVA fibers & 25 & 25 & 25 & 24.8 & $\mathrm{~m}-\%$ \\
Activated carbon fibers & 15 & 15 & 15 & 14.9 & $\mathrm{~m}-\%$ \\
Glass nanofibers & & 10 & 9 & 9.9 & $\mathrm{~m}-\%$ \\
Ag nanofibers & & & - & $\mathrm{m}-\%$ & $\mathrm{~m}-\%$ \\
Ag nanoparticles & & & & 1 & \\
\hline
\end{tabular}


mass-size distribution was obtained through the number-size and effective density measurements described above.

\section{Methanol Adsorption}

The performance of the ACF in the filters against VOCs were tested with the adsorption of methanol, which is a typical VOC. Measurements were done with a similar apparatus as described by Kumita et al. (2018); the measurement setup is shown in Fig. 1(b). The apparatus consists of three temperature-controlled chambers: one with liquid methanol, one for storing the methanol vapor and the last with the tested sample in it. The adsorption of methanol was measured as a function of relative pressure, which is a proportion of the saturated vapor pressure of methanol at $30^{\circ} \mathrm{C}$. First, a vacuum was pulled to the second and third chambers to remove all of the present water. Then, increasing pressures of methanol vapor were introduced to the second chamber. At each pressure point, the sample is allowed to adsorb the VOC vapor by connecting the second and third chambers together. When the pressure stops decreasing, the value is logged. For comparison, the amount of adsorbed methanol, $q$, as a function of relative pressure for pure ACF was used as reference. The same apparatus was also used for quantifying the volume of each of the filter media to know the space occupied by the filter in the third chamber and thus the pressure drop caused by the connection of the second and third chamber. Additionally, by knowing the dimensions of the filter disk, mass fractions of the utilized filter fibers and the total mass of the filters, packing density $(\alpha)$ values can be calculated. The relevant values are shown in Table 2 and were used for the calculation of the theoretical particle penetration, utilizing the theory described by Choi et al. (2017). An overview of the theory is given in the next section.

\section{Particle Penetration}

The theoretical penetration, $P$, of the fiber filters can be calculated, assuming monodisperse fiber diameters, $d_{f}$, with the following equation:

$$
P=\exp \left\{-\frac{4}{\pi} \frac{\alpha}{(1-\alpha)} \frac{L}{\bar{d}_{f}} \frac{\eta}{\delta}\right\},
$$

where $L$ is the filter thickness, $\delta$ is an inhomogeneity factor

(a)
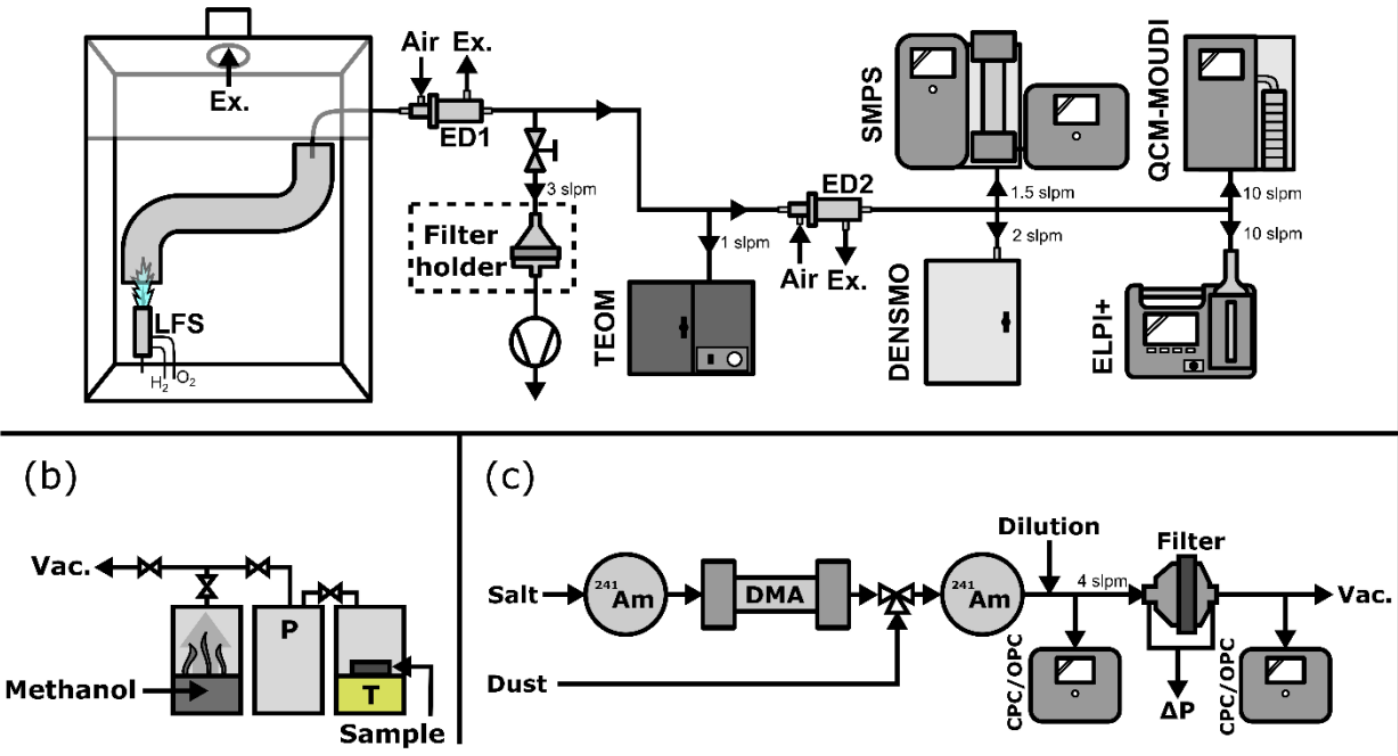

Fig. 1. Experimental setups for (a) the nanoparticle coating process, (b) the methanol adsorption measurements and (c) the particle penetration measurements. In the particle penetration measurements, two particle sources were used: Salt was generated with a tubular furnace and dust particles with a brush generator.

Table 2. Specifications relating to the filter structure and performance.

\begin{tabular}{llllll}
\hline & & Microfibers & Nanofibers & Ag-fibers \\
& & $\mathrm{F} 0$ & $\mathrm{~F} 1$ & $\mathrm{~F} 2$ & \\
\hline Thickness & $\mathrm{L}$ & 0.65 & 0.51 & 0.48 & $\mathrm{~mm}$ \\
Diameter & & 47 & 47 & 47 & $\mathrm{~mm}$ \\
Area density & $\mathrm{W}$ & 54.4 & 63.7 & 62.7 & $\mathrm{~g} \mathrm{~m}^{-2}$ \\
Pressure drop (at $3.8 \mathrm{~cm} \mathrm{~s}^{-1}$ ) & $\Delta \mathrm{P}$ & 4.5 & 35.1 & 33.0 & $\mathrm{~Pa}$ \\
Pressure drop, theoretical & $\Delta \mathrm{P}_{\mathrm{t}}$ & 10.2 & 38.4 & 43.1 & $\mathrm{~Pa}$ \\
Packing density & $\alpha$ & 0.145 & 0.277 & 0.314 & \\
\hline
\end{tabular}


defined as the ratio between the theoretical and the measured pressure drops and $\eta$ is the combined collection efficiency of diffusion and interception collection mechanisms, as well as their interaction term. As the prepared filters contained both microfibers and nanofibers, it is considered a mixed fiber filter. The effect the different collection mechanisms have can be first considered separately for these different diameter fibers and then combined later. This penetration is calculated for the micro- and nanofibers separately and then combined with the following equation:

$P=\exp \left(\ln P_{M}+\ln P_{N}\right)$

where the subscripts denote the contributions of micro- $M$ and nano- $N$ fibers.

The particle penetration of the filters was tested by introducing particles in the range of $30 \mathrm{~nm}-7 \mu \mathrm{m}$ and measuring the downstream and upstream particle concentrations with a condensation particle counter (CPC; Model 3775; TSI Inc.) or an optical particle counter (OPC; Model 3080; TSI Inc.) based on their measurement ranges. Salt $(\mathrm{NaCl})$ produced by a tubular furnace was used to produce the smaller particles and a fluidized bed aerosol generator (Model 3080; Kanomax) was used to disperse JIS Class 11 test powder (SP3-3, Association of Powder Process Industry and Engineering), generating the larger end of the size range. The test setup is shown in Fig. 1(c), where the whole dust number size distribution is filtered by the test filter and the salt particles are size selected so that only a monodisperse distribution is being filtered at a time. The face velocity of the aerosol penetrating the filter was kept at $3.8 \mathrm{~cm} \mathrm{~s}^{-1}$.

\section{Antibacterial Testing}

Antibacterial capabilities of the prepared filters were tested with a touch test method, described in the work by
Gunell et al. (2017), against gram-negative E. coli (ATCC 25922) and gram-positive $S$. aureus (methicillin-susceptible strain, ATCC 29213) on blood agar plates (Trypticase Soy Agar with 5\% Sheep Blood [II]; BD). Here, the reference sample was the filter F1, which contains all the other kinds of fibers excluding silver, which was compared against F2 and F3. The bacteria were incubated on the samples at room temperature for 24 and $48 \mathrm{~h}$, after which the samples were placed on the blood agar plates to transfer any viable bacteria to be calculated the next day. The bacterial growth was estimated based on the amount of colony forming units (CFUs) in the range of $0-3$, as per the method definition: 0 meaning no growth and going up to 3, meaning good growth with $>100$ colonies.

\section{RESULTS AND DISCUSSION}

\section{Coating Mass Loading}

The results from the coating process characterization can be seen in Fig. 2, where the results have been split into (a) number and (b) mass concentration plots. The produced silver particles have a count mean diameter (CMD) of $43 \mathrm{~nm}$ based on the SMPS measurement and aerodynamic mean diameter of $258 \mathrm{~nm}$ based on the ELPI+ measurement. Using Eq. (1), an effective density value of $10.2 \mathrm{~g} \mathrm{~cm}^{-3}$ can be calculated, which tells us that the silver is mostly nonagglomerated and close to spherical in shape. Information about the measured silver nanoparticle number size distribution was needed in the estimation of what part of it is deposited on the filter fibers. To achieve this, the information was combined with the filter particle collection efficiency.

The mass measurements made with the TEOM and QCM-MOUDI give similar results on the total mass concentration with measured values of $15.6 \mathrm{mg} \mathrm{m}^{-3}$ and $16.1 \mathrm{mg} \mathrm{m}^{-3}$, respectively. This mass concentration is determined after the first ejector diluter, after which the (a)

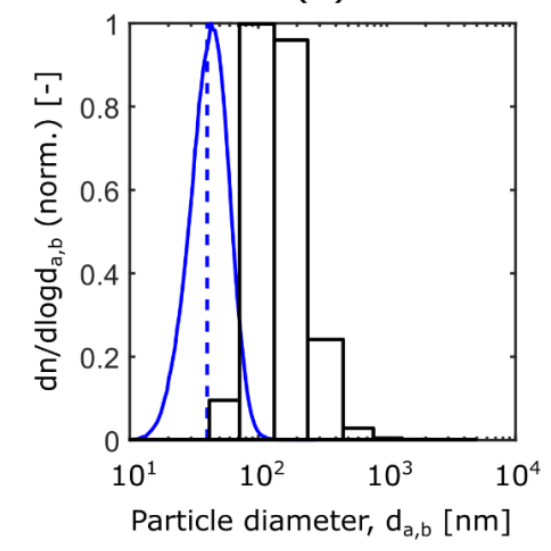

(b)

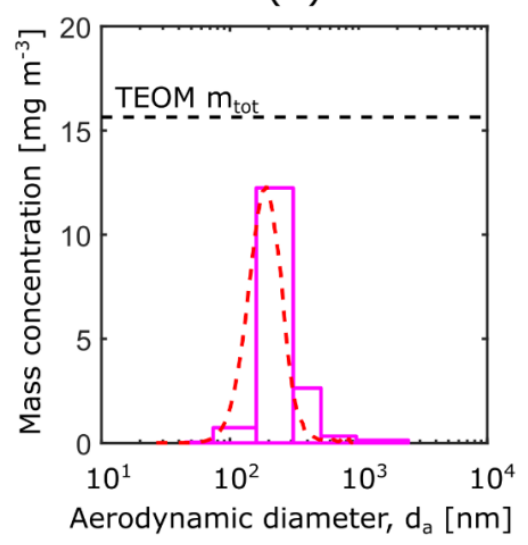

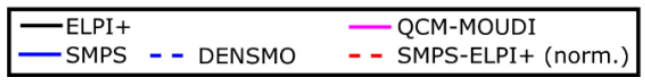

Fig. 2. Aerosol measurements for the mass loading estimation. Left side shows the SMPS- and ELPI+-measured number size distributions of the coating particles as a function of mobility size and aerodynamic size, respectively. Dashed line shows the time averaged CMD value from DENSMO. Right side shows the mass size distribution from QCM-MOUDI and a normalized one from the SMPS-ELPI+ estimation, and the total mass concentration from TEOM. 
aerosol is directed to the filter holder; thus, the mass concentration flowing into the filter holder is the same. Another way to estimate the mass distribution is to use the measurement results from the SMPS and ELPI+. This calculated distribution is shown normalized in Fig. 2(b) and yields a total mass concentration of $14.5 \mathrm{mg} \mathrm{m}^{-3}$. In order to achieve the $1 \mathrm{~m}-\%$ coating of silver, approximately a 25 min coating time was required. To have more even distribution of the silver inside the filter media, the coating was done in two 12.5 min parts, one for each side of the filter media.

Given that the two ejector diluters both have a dilution factor of about 8 , an estimate can be made of the dilution factor of the residence tube. The residence tube affects the total mass of the produced silver, with the used parameters, by a factor of 125 . This dilution was caused by diffusion and possible impaction of precursor droplets, as well as the convective air flow of the surrounding air. The high flow rate of the generation method enables scaling up, where more of the produced silver could be sampled with parallelizing multiple inlets or just scaling the current one.

The process was also monitored with DENSMO so that the stability of the mobility diameter, aerodynamic diameter and effective density could be observed in real time during the coating. DENSMO measured the CMD to be $40 \mathrm{~nm}$ and the effective density value as $11.2 \mathrm{~g} \mathrm{~cm}^{-3}$, which are both within the measurement range from the $43 \mathrm{~nm}$ measured by the SMPS and the effective density of $10.2 \mathrm{~g} \mathrm{~cm}^{-3}$ calculated from the SMPS and ELPI+ measurements.

\section{SEM Micrography}

To have a better understanding of the structure and distribution of the fibers in the produced filter media, as well as the result of the nanoparticle coating, a scanning electron microscope (SEM; ULTRA plus; ZEISS) was used. Fig. 3 shows the as-prepared filters F0 and F1, and the coated filter F3. F2 is not shown, due to having identical appearance to F1. The low count and the narrow diameter of the silver nanofibers makes them difficult to find with the used SEM. The three different base materials can be clearly identified from Fig. 3(a) and 3(d) by their distinct interactions with the electron beam. The introduction of the glass nanofibers can be seen in Figs. 3(b) and 3(e) to fill the voids left by the microfibers, which can be attributed to increase the particle interception efficiency. Lastly, in Figs. 3(c) and 3(f), the silver coating can be seen to be distributed homogeneously, at least in the lateral direction.

\section{Performance of the Filter Functionality}

The methanol adsorption isotherm at $30^{\circ} \mathrm{C}$ as a function of the relative pressure can be seen in Fig. 4. The values of adsorbed amounts are similar to those reported by Kumita et al. (2017). The amount adsorbed here is related to the mass of ACF in the filter media. The measurements show that the inclusion of other fibers in the filter does not inhibit the adsorption potential of ACF when compared to the bulk material. This means that the amount of ACF can be tailored to suit a specific need.

Particle penetration through filters F0, F1 and F2 are presented in Fig. 5(a). The face velocity during the measurements was kept at $3.8 \mathrm{~cm} \mathrm{~s}^{-1}$. By introducing glass nanofibers into the filter media, a clear reduction can be seen in the penetration, as is to be expected. However, substituting some of the glass nanofibers with silver nanofibers increases the penetration slightly. This is due to the reduction in the count of nanofibers in the filter media, caused by the higher density of silver compared to glass. In essence, the same mass of nanofibers contains less fibers overall. Theoretical particle penetration curves were calculated based on Eq. (2) with packing density values of $0.145,0.277$ and 0.311 , and initial pressure drops of 4.5, 35.1 and 33.0 Pa for filters F0, F1 and F2 respectively. Additionally, the efficiency can be increased by stacking the prepared filter media, as is typically done. The quality factors for these filters are shown in Fig. 5(b). There is no significant improvement on the performance for nanoscale particles, as the increase of the collection efficiency has been achieved with the cost of increased pressure drop. For particle size ranges closer to a micrometer, the interception is increased significantly more, which can also be seen in the increase in the quality factor.

Antibacterial activity against $S$. aureus and E. coli is shown in Fig. 6, where an image set of agar plates covering the four test cases are displayed. In some of the test cases the handling and cutting of the filter media has resulted in loose fibers, which then can be seen on the agar plates. These should not be mistaken for bacterial growths. Averaged
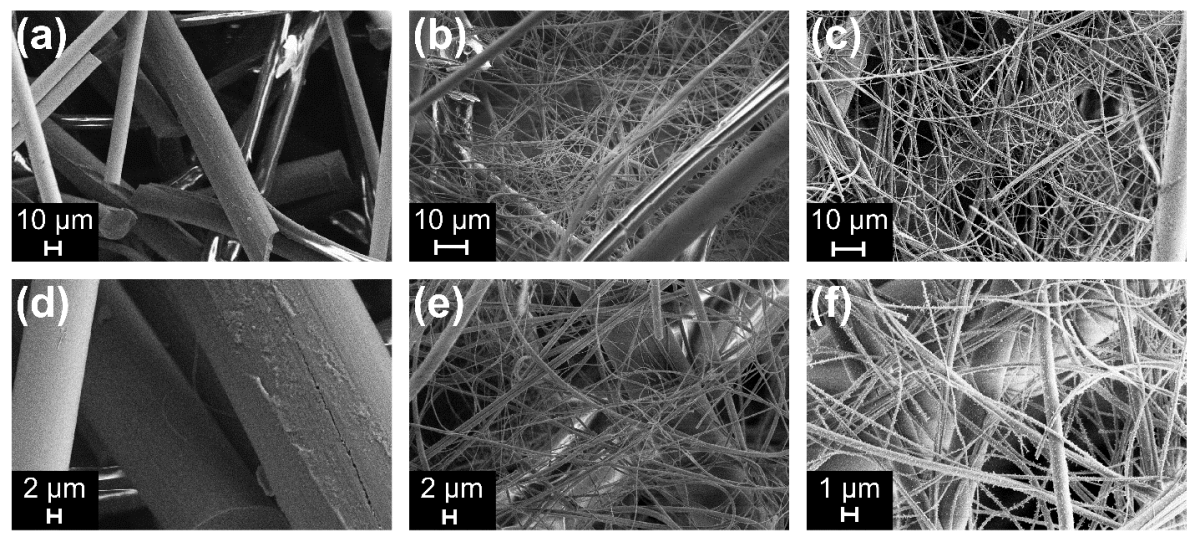

Fig. 3. SEM-micrographs from (a, d) F0, (b, e) F1 and (c, f) F3 filter materials, with two magnifications. 


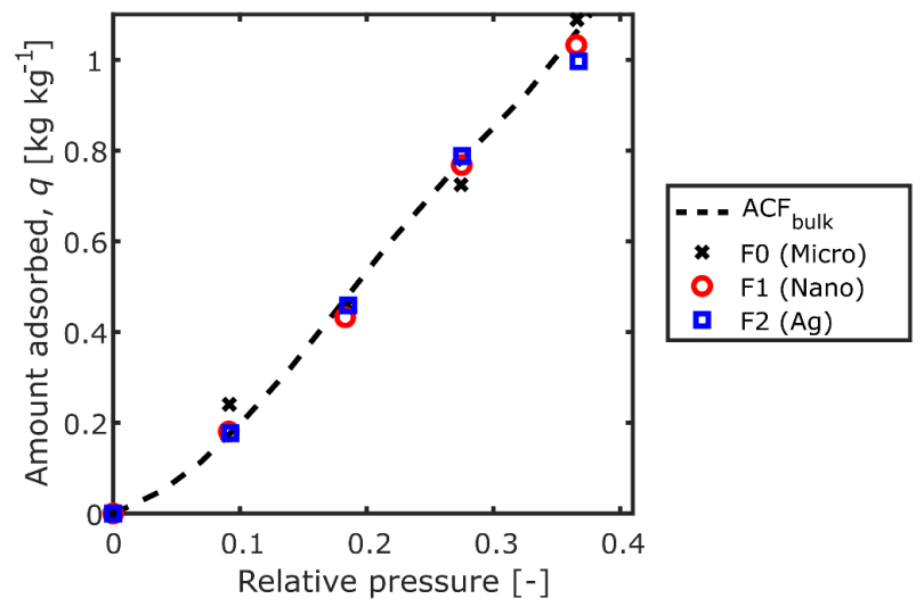

Fig. 4. Results from the methanol adsorption filter tests.

(a)

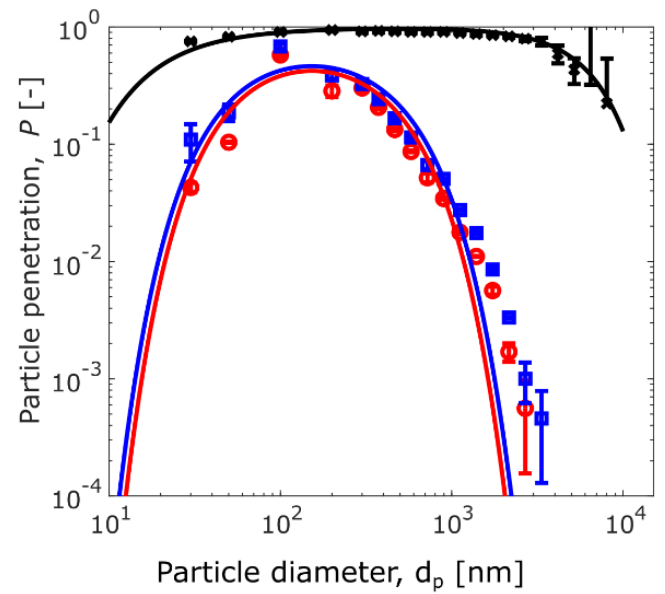

(b)

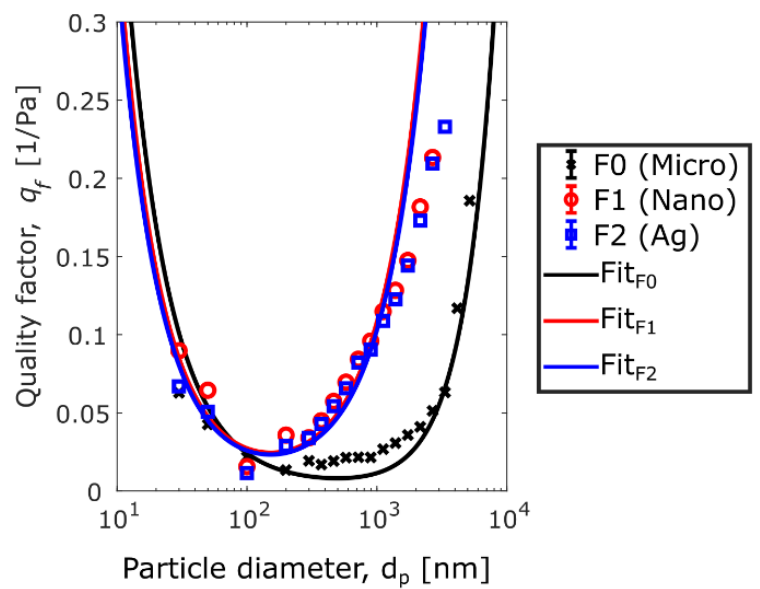

Fig. 5. Filtration performance of the prepared filters: (a) the particle penetration and (b) the quality factors.

set of data from the bacterial growths is shown in Fig. 7. In all of the cases, the bacterial growth was not inhibited by the F1 reference sample, but the addition of nanoscale silver can clearly be seen to increase the antibacterial activity. Both the silver nanofibers and nanoparticles are very effective against $E$. coli, effectively blocking the growth completely on all the studied cases. Minimal growth was found on one silver fiber containing filter after $24 \mathrm{~h}$ of incubation. $S$. aureus, on the other hand, is much more resistant against the silver present in the filters. Additionally, the silver particles were slightly more effective as an antibacterial additive in the filters when compared to the nanofibers. Relying on the measurements made in this work, it is difficult to say exactly what causes this disparity between these two materials. It could be their morphologies, being the most obvious difference, or the distribution of silver inside the filter media. In either case, more measurements would be needed to reveal the actual mechanism. Overall, the comparable activity of these materials is probably due to the same mass loading of silver in the filter media. In the work of Gunell et al. (2017), the amount of silver needed to inhibit the growth of these same bacteria was studied, and based on their results, by increasing the amount of silver in the filter media of this work, the growth of $S$. aureus could also be suppressed.

\section{CONCLUSIONS}

Antibacterial fiber filters containing activated carbon fibers were fabricated using nanoscale silver and tested for particle penetration, methanol adsorption and bacterial growth inhibition. The silver was introduced into the filter media either during the filter fabrication process as silver nanofiber content or after the filter fabrication process as an LFS-generated nanoparticle coating. The performance in terms of particle penetration and methanol adsorption agreed well with the theory and bulk reference, respectively, despite the filter media consisting of mixed materials. Antibacterial activity was successfully generated by both methods. However, the silver nanofibers showed slightly higher activity than the nanoparticles, which cannot be attributed to inconsistent mass loading of the silver between the filter media, as the coating process was monitored rigorously with a wide range of instruments to prevent 
(a)

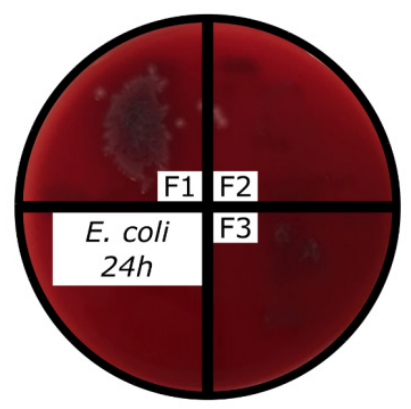

(c)

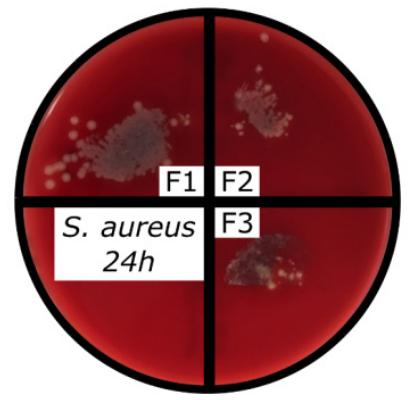

(b)

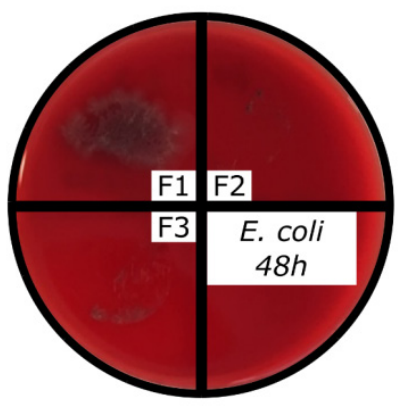

(d)

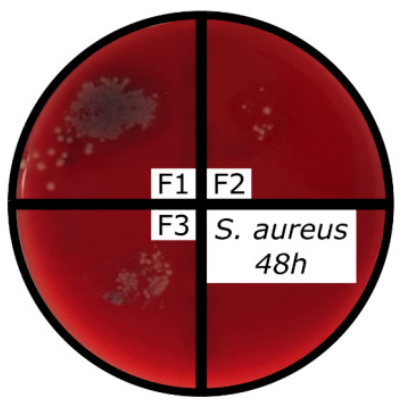

Fig. 6. Images of all four test cases: (a) E. coli (24 h), (b) E. coli (48 h), (c) S. aureus (24 h) and (d) S. aureus (48 h), which show the bacterial growths for the reference filter (F1) and the two antibacterial filters (F2 and F3).

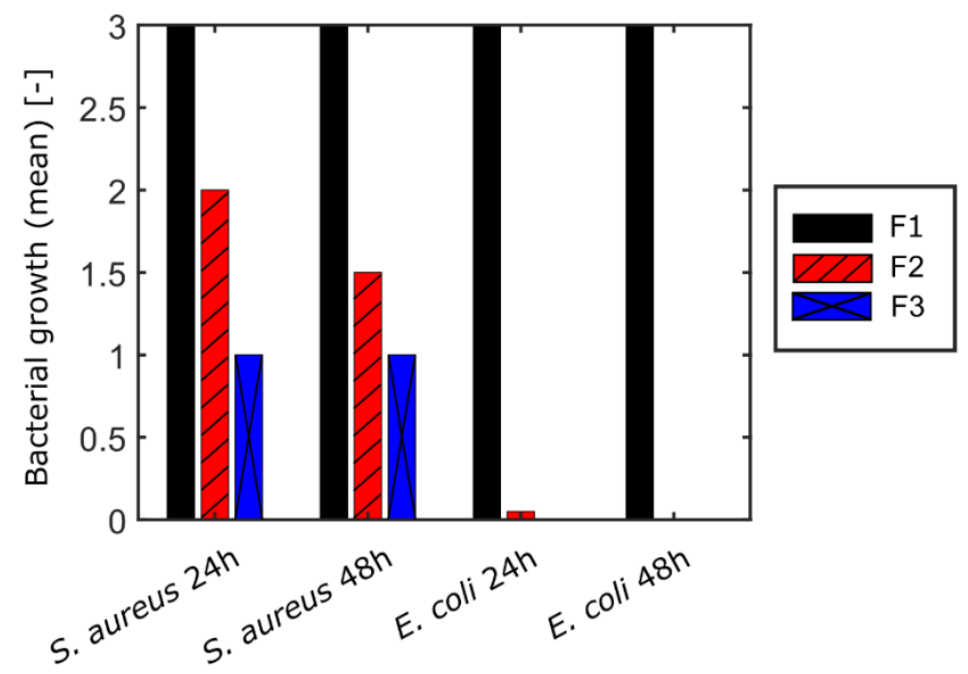

Fig. 7. The overall antibacterial activity of the prepared filter materials.

such a discrepancy. Regardless, with either method, the resultant antibacterial filters can be tuned to specific needs by adjusting the thickness of the filter or the mass fraction of added ACF or silver. Furthermore, the coating process can be applied to existing filters that are already in use, for example, in HVAC systems, which would be the next logical step.

\section{ACKNOWLEDGMENTS}

Paxton Juuti acknowledges TUT's graduate school and the Emil Aaltonen Foundation for financial support. The authors would also like to thank Miki Tavast for the QCMMOUDI and Dr. Mari Honkanen for the SEM imaging.

\section{REFERENCES}

Brobbey, K., Haapanen, J., Gunell, M., Mäkelä, J., Eerola, E., Toivakka, M. and Saarinen, J. (2017). One-step flame synthesis of silver nanoparticles for roll-to-roll production of antibacterial paper. Appl. Surf. Sci. 420: 558-565.

Choi, H.J., Kumita, M., Hayashi, S., Yuasa, H., Kamiyama, M., Seto, T., Tsai, C.J. and Otani, Y. (2017). 
Filtration properties of nanofiber/microfiber mixed filter and prediction of its performance. Aerosol Air Qual. Res. 17: 1052-1062.

Daisey, J., Angell, W. and Apte, M. (2003). Indoor air quality, ventilation and health symptoms in schools: An analysis of existing information. Indoor Air 13: 53-64.

Forthomme, A., Joubert, A., Andrès, Y., Simon, X., Duquenne, P., Bemer, D. and Coq, L.L. (2014). Microbial aerosol filtration: Growth and release of a bacteria-fungi consortium collected by fibrous filters in different operating conditions. J. Aerosol Sci. 72: 32-46.

Gordon, T., Perlstein, B., Houbara, O., Felner, I., Banin, E. and Margel, S. (2011). Synthesis and characterization of zinc/iron oxide composite nanoparticles and their antibacterial properties. Colloids Surf., A 374: 1-8.

Gunell, M., Haapanen, J., Brobbey, K., Saarinen, J., Toivakka, M., Mäkelä, J.M., Huovinen, P. and Eerola, E. (2017). Antimicrobial characterization of silver nanoparticle-coated surfaces by "touch test" method. Nanotechnol. Sci. Appl.10: 137-145.

Haapanen, J., Aromaa, M., Teisala, H., Juuti, P., Tuominen, M., Sillanpää, M., Stepien, M., Saarinen, J.J., Toivakka, M., Kuusipalo, J. and Mäkelä, J. (2018). On the limit of superhydrophobicity: Defining the minimum amount of $\mathrm{TiO}_{2}$ nanoparticle coating. Mater. Res. Express 14: 035004.

Harra, J., Kujanpää, S., Haapanen, J., Juuti, P., Mäkelä, J. M., Hyvärinen, L. and Honkanen, M. (2017). Aerosol analysis of residual and nanoparticle fractions from spray pyrolysis of poorly volatile precursors. AIChE J. 63: 881-892.

Hassan, I., Parkin, I., Nair, S. and Carmalt, C. (2014). Antimicrobial activity if copper and copper(I) oxide thin films deposited via aerosol-assisted CVD. J. Mater. Chem. B 2: 2855.

Jones, A. (1999). Indoor air quality and health. Atmos. Environ. 33:4535-4564.

Jung, J., Hwang, G., Lee, J. and Bae, G. (2011). Preparation of airborne $\mathrm{Ag} / \mathrm{CNT}$ hybrid nanoparticles using an aerosol process and their application to antimicrobial air filtration. Langmuir 27: 10256-10264.

Juuti, P., Arffman, A., Rostedt, A., Harra, J., Mäkelä, J. and Keskinen, J. (2016). Real-time effective density monitor (DENSMO) for aerosol nanoparticle production. Aerosol Sci. Technol. 50:487-496.

Juuti, P., Haapanen, J., Stenroos, C., Niemelä-Anttonen, H., Harra, J., Koivuluoto, H., Teisala, H., Lahti, J., Tuominen, M., Kuusipalo, J., Vuoristo, P. and Mäkelä, J.M. (2017). Achieving a slippery, liquid-infused porous surface with anti-icing properties by direct deposition of flame synthesized aerosol nanoparticles on a thermally fragile substrate. Appl. Phys. Lett. 110: 161603.

Kulkarni, P., Baron, P. and Willeke, K. (2011). Aerosol measurement: Principles, techniques, and applications.
Wiley.

Kumita, M., Yamawaki, N., Shinohara, K., Higashi, H., Kodama, A., Kobayashi, N., Seto, T. and Otani, Y. (2018). Methanol adsorption behaviors of compressionmolded activated carbon fiber with PTFE. Int. J. Refrig. 94: 127-135.

Mäkelä, J.M., Haapanen, J., Harra, J., Juuti, P. and Kujanpää, S. (2017). Liquid flame spray - A hydrogenoxygen flame based method for nanoparticle synthesis and functional nanocoatings. Kona Powder Part. J. 34: 141-154.

Möritz, M., Peters, H., Nipko, B. and Rüden, H. (2001). Capability of air filters to retain airborne bacteria and molds in heating, ventilating and air-conditioning (HVAC) systems. Int. J. Hyg. Environ. Health 203: 401-409.

Oya, A., Yoshida, S., Abe, Y., Itzuka, T. and Makiyama, N. (1993). Antibacterial activated carbon fiber derived from phenolic resin containing silver nitrate. Carbon 32: $71-73$.

Rumchev, K.B., Spickett, J.T., Bulsara, M.K., Phillips, M.R. and Stick, S.M. (2002). Domestic exposure to formaldehyde significantly increases the risk of asthma in young children. Eur. Respir J. 20: 403-408.

Saad, S. (2003). Integrated environmental management for hospitals. Indoor Built Environ. 12: 93-98.

Sorvali, M., Vuori, L., Pudas, M., Haapanen, J., Mahlberg, R., Ronkainen, H., Honkanen, M., Valden, M. and Mäkelä, J.M. (2018). Fabrication of ultrathin multilayered superomniphobic nanocoatings by liquid flame spray, atomic layer deposition, and silanization. Nanotechnology 29:185708

Sotiriou, G. and Pratsinis, S. (2010). Antibacterial activity of nanosilver ions and particles. Environ. Sci. Technol. 44: 5649-5654.

Teisala, H., Geyer, F., Haapanen, J., Juuti, P., Mäkelä, J.M., Vollmer, D. and Butt, H.J. (2018). Ultrafast processing of hierarchical nanotexture for a transparent superamphiphobic coating with extremely low roll-off angle and high impalement pressure. Adv. Mater. 30: 1706529.

Tikkanen, J., Gross, K., Berndt, C., Pitkänen, A., Keskinen, J., Raghu, S., Rajala, M. and Karthikeyan, J. (1997). Characteristics of the liquid spray process. Surf. Coat. Technol. 90: 210-216.

Yoon, K., Byeon, J., Park, C. and Hwang, J. (2008). Antimicrobial effect of silver particles on bacterial contamination of activated carbon fibers. Environ. Sci. Technol. 42: 1251-1255.

Received for review, December 21, 2018 Revised, April 25, 2019 Accepted, June 24, 2019 